\title{
Benign concentric annular macular dystrophy
}

INSERM

\section{Source}

INSERM. (1999). Orphanet: an online rare disease and orphan drug data base. Benign concentric annular macular dystrophy. ORPHA:251287

Benign concentric annular macular dystrophy (BCAMD) is a progressive autosomal dominant macular dystrophy characterized by parafoveal hypopigmentation followed by a retinitis pigmentosa-like phenotype (nyctalopia and peripheral vision loss) with a bulls eye configuration. 